\title{
Frequência de anemia: uma comparação entre gestantes adolescentes e adultas
}

\author{
Frequency of anemia: a comparison between adolescent and adult pregnant women
}

Frecuencia de anemia: una comparación entre mujeres embarazadas adolescentes y adultas

Luísa de Carvalho Mares Guia' ${ }^{1}$, Emille Meira Lessa1, Tamara Lacerda Mesquita1', Larissa Carvalho de Queiroz ${ }^{1}$, Isadora Vitor de Oliveira ${ }^{1}$, Thaís Melo Pereira ${ }^{2}$, Gabriela Barbosa Lima ${ }^{2}$, Gabriel Duque Pannain $^{3}$, Clara de Oliveira Menon ${ }^{2}$, Juliana Barroso Zimmermmann ${ }^{1,2 *}$.

\section{RESUMO}

Objetivo: Avaliar a frequência de anemia em gestantes adolescentes e adultas. Métodos: Tratou-se de uma coorte histórica que estudou dados de 1414 gestantes, sendo 293 adolescentes e 1121 adultas. Resultados: A anemia ferropriva foi identificada em $98 \%$ e a macrocítica em $2 \%$ dos casos. A média de hemoglobina nas gestantes adolescentes foi de $11,7( \pm 1,37)$ no primeiro, $11,30( \pm 1,09)$ no segundo e $11,50( \pm 1,52)$ no terceiro trimestre. Já nas adultas, observou-se $12,10( \pm 1,25), 11,50( \pm 1,12)$ e $11,80( \pm 1,40)$ respectivamente. A comparação entre os dois grupos revelou $p<0,001$ para o primeiro trimestre, $p=0,01$ no segundo e $p=0,05$ no terceiro. A frequência de anemia foi calculada, sendo esta de $20 \%$ no primeiro, $29,4 \%$ no segundo e $18,2 \%$ no terceiro trimestre. A anemia foi mais frequente em gestantes adolescentes $(R R=1,93 ; I C=1,40-2,66$; $p<0,001)$, no primeiro trimestre. Após a aplicação do modelo de regressão, a renda familiar $(p<0,001)$ e a adolescência $(p=0,003)$ foram as variáveis significativas associadas à anemia. Conclusão: Conclui-se que a anemia teve maior frequência nas adolescentes, estando associada à baixa renda e à idade. Entretanto, esta não se relacionou ao baixo peso fetal, infecção urinária e/ou corrimento vaginal, evidenciando que um prénatal adequado pode modificar os desfechos desfavoráveis materno-fetais.

Palavras-chave: Anemia, Cuidado pré-natal, Adolescência, Complicações na gravidez.

\begin{abstract}
Objective: To assess the frequency of anemia in pregnant adolescents and adults. Methods: This was a historical cohort that studied data from 1414 pregnant women, 293 adolescents and 1121 adults. Results: Iron deficiency anemia was identified in $98 \%$ and macrocytic in $2 \%$ of cases. The mean hemoglobin in pregnant adolescents was $11.7( \pm 1.37)$ in the first, $11.30( \pm 1.09)$ in the second and $11.50( \pm 1.52)$ in the third trimester. In adults, $12.10( \pm 1.25), 11.50( \pm 1.12)$ and $11.80( \pm 1.40)$ respectively were observed. The comparison between the two groups revealed $p<0.001$ for the first trimester, $p=0.01$ in the second trimester and $p=0.05$ in the third trimester. The frequency of anemia was calculated, being $20 \%$ in the first, $29.4 \%$ in the second and $18.2 \%$ in the third trimester. Anemia was more frequent in pregnant adolescents $(R R=1.93 ; \mathrm{Cl}=1.40-2.66 ; p<0.001)$ in the first trimester. After applying the regression model, family income $(p<0.001)$ and adolescence $(p=0.003)$ were the significant variables associated with anemia. Conclusion: It is concluded that anemia was more frequent in adolescents, being associated with low income and age. However, this was not related to low fetal weight, urinary infection and/or vaginal discharge, showing that adequate prenatal care can modify unfavorable maternal-fetal outcomes.
\end{abstract}

Keywords: Anemia, Prenatal care, Adolescents, Pregnancy complications.

\section{RESUMEN}

Objetivo: Evaluar la frecuencia de anemia en adolescentes y adultas embarazadas. Métodos: Se trata de una cohorte histórica que estudió datos de 1414 mujeres embarazadas, 293 adolescentes y 1121 adultos. Resultados: Se identificó anemia ferropénica en el $98 \%$ y macrocítica en el $2 \%$ de los casos. La hemoglobina media en adolescentes embarazadas fue $11,7( \pm 1,37)$ en el primero, $11,30( \pm 1,09)$ en el segundo y $11,50( \pm$ $1,52)$ en el tercer trimestre. En adultos se observaron $12,10( \pm 1,25), 11,50( \pm 1,12)$ y $11,80( \pm 1,40)$

\footnotetext{
${ }^{1}$ Faculdade de Medicina de Barbacena (FUNJOBE), Barbacena - MG. *E-mail: julianabz@uol.com.br

${ }^{2}$ Faculdade de Medicina da UFJF, Juiz de Fora - MG

${ }^{3}$ Hospital Universitário (EBSERH), Juiz de Fora - MG.
}

SUBMETIDO EM: 7/2021

ACEITO EM: 7/2021

PUBLICADO EM: 7/2021 
respectivamente. La comparación entre los dos grupos reveló $p<0.001$ para el primer trimestre, $p=0.01$ en el segundo yp $=0.05$ en el tercero. Se calculó la frecuencia de anemia, siendo $20 \%$ en el primero, $29,4 \%$ en el segundo y $18,2 \%$ en el tercer trimestre. La anemia fue más frecuente en las adolescentes embarazadas $(R R=1,93 ; I C=1,40-2,66 ; p<0,001)$ en el primer trimestre. Tras aplicar el modelo de regresión, el ingreso familiar $(p<0,001)$ y la adolescencia $(p=0,003)$ fueron las variables significativas asociadas a la anemia. Conclusión: Se concluye que la anemia fue más frecuente en adolescentes, asociándose con bajos ingresos y edad. Sin embargo, esto no se relacionó con bajo peso fetal, infección urinaria y / o flujo vaginal, lo que demuestra que una atención prenatal adecuada puede modificar los resultados materno-fetales desfavorables.

Palabras clave: Anemia, Cuidado prenatal, Adolescentes, Complicaciones del embarazo.

\section{INTRODUÇÃO}

A Organização Mundial da Saúde (OMS), define anemia quando a hemoglobina está baixa, devido à deficiência de nutrientes essenciais, independente da origem desta carência (OMS, 2013). Dessa forma, pode-se dizer que anemia é uma condição em que há redução no número de eritrócitos por unidade de volume sanguíneo ou do conteúdo de hemoglobina ou até mesmo quando a concentração de hemoglobina estiver abaixo de 95\% para população saudável de referência (homens, mulheres ou crianças) agrupadas por faixa etária (BRASIL, 2015).

A gestação normal caracteriza-se por uma série de ajustes, sejam fisiológicos ou anatômicos que determinam alterações no organismo materno, como a composição do sangue circulante. O conhecimento dessas mudanças é necessário para uma adequada avaliação do que deveria ser considerado doença na gravidez (CHURCHIL D, et al., 2019).

Na gestação, há uma elevação do volume sanguíneo total pelo aumento do volume plasmático ( $40 \%$ a $50 \%$ ), da massa total de eritrócitos e dos leucócitos circulantes. O volume plasmático eleva-se progressivamente a partir da sexta semana de gestação, expande-se durante o segundo trimestre, com pico em torno da $24^{\underline{a}}$ semana, estabilizando-se até o final da gestação ( $32^{\underline{a}}$ a $34^{\underline{a}}$ semanas) (CHURCHIL D, et al., 2019). Dessa forma, ocorre um aumento mais acentuado do volume plasmático quando comparado com a massa eritrocitária e, por isso, os indicadores hematológicos (contagem de células vermelhas, níveis de hemoglobina $(\mathrm{Hb})$ e hematócrito $(\mathrm{HTC})$ se reduzem a partir do segundo trimestre da gestação, caracterizando a anemia fisiológica da gravidez, explicada pela desproporção entre o aumento da massa eritrocitária e do plasma sanguíneo (GANDHI MH e GUPTA VK, 2021).

A anemia é um sinal-sintoma de uma variedade de situações, incluindo grande perda de sangue, destruição excessiva de células sanguíneas ou diminuição de sua formação. Uma ampla variedade de fatores pode causar anemia, incluindo doenças hereditárias, deficiências nutricionais (déficit de ácido fólico, vitamina B12 e ferro), hemorragia, infecções, doenças crônicas e neoplasias (JUGHA VT, et al., 2021; PATEL P e BALANCHIVADZE N, 2021).

Dentre os problemas nutricionais destaca-se a anemia ferropriva, que determina complicações tanto para a gestante quanto para o concepto, sendo associada a risco de morbimortalidade materno-fetal (SUN D, et al., 2017; PARKS S, et al., 2019). Quando se avalia anemia em adolescentes, acredita-se que essa incremente o baixo peso ao nascer, crescimento intrauterino restrito e até baixo índice de apgar (KARAÇAM Z, et al., 2021; BOMFIM VVBS, et al., 2020).

A gestação na adolescência, é um problema de saúde pública, já que muitas engravidam de forma indesejada. A perpetuação da pobreza que acontece com o abandono escolar e a ausência de profissionalização, colocando-as fora do mercado de trabalho, associado à dificuldade de estrutura familiar nessa faixa etária, colocam essas pacientes em grupos vulneráveis e de risco para uma série de complicações. Esse estudo identificou que mães de 10 a 14 anos tinham maior probabilidade de serem solteiras, rurais, indígenas ou negras e tinham menos acesso a cuidados de saúde, correndo maior risco de perda gestacional quando são pobres e permanecem pobres (JARAMILLO-MEJIA MC e CHERNICHOVSKY D, 2019). 
A anemia é um desses problemas, já que as reservas de ferro diminuem ao longo da gravidez e, nesse caso, a demanda está aumentada, não apenas pela presença do feto, mas também pelo próprio organismo da adolescente, ainda em desenvolvimento. Sendo assim, muitos autores têm relatado prevalências elevadas de anemia em gestantes adolescentes em outras regiões de nosso país, com variações de 40 a $50 \%$ (ANDRADE TR e CAIXETA BT, 2018).

Dessa forma a anemia é um problema relevante e descrito na literatura médica mundial e, por isso, conhecer sua frequência e fatores associados são importantes para estratégia de profilaxia e tratamento, especialmente considerando a heterogeneidade do Brasil. Baseado no exposto, objetivou-se avaliar a frequência de anemia nutricional em gestantes bem como fazer uma comparação entre gestantes adultas e adolescentes e associação com dados epidemiológicos e clínicos maternos e fetais.

\section{MÉTODOS}

Tratou-se de uma coorte histórica onde foram estudados dados de pacientes de baixo risco atendidas pelo serviço de Obstetrícia da Faculdade de Medicina no período de setembro de 2008 a setembro de 2019. Avaliou-se dados de 1414 gestantes, sendo 293 adolescentes e 1121 não adolescentes (adultas).

A utilização de prontuários dos dois serviços foi possível porque eles usam prontuários padronizados disponibilizados pela Febrasgo e tem o mesmo coordenador na equipe, o que permitiu que os dados fossem avaliados de forma homogênea. Dados da anamnese (idade, gesta, partos prévios, abortos, ganho de peso no pré-natal) foram anotados, bem como a identificação de fluxo genital patológico, infecção urinária, sífilis, altura uterina e peso fetal estimado no último trimestre de gestação.

Todas as pacientes receberam dosagem de 40 a $60 \mathrm{mg} /$ dia de sulfato ferroso, conforme orientações do Ministério da Saúde a qual os serviços estão vinculados. Foram, então, incluídos dados de gestantes de ambos os serviços no período proposto e excluídos aqueles incompletos e os casos de anemias hereditárias. As pacientes foram acompanhadas mensalmente até 36 semanas e, a seguir, semanalmente até o parto.

Considerou-se apenas os casos de anemia nutricional, seja pelo déficit de ferritina $(<15 \mathrm{mcg} / \mathrm{dl})$, ácido fólico $(<4 \mathrm{ng} / \mathrm{ml})$ ou vitamina B12 $(<211 \mathrm{pg} / \mathrm{ml})$. A anemia ferropriva foi diagnosticada quando o nível de hemoglobina estava abaixo de $11 \mathrm{mg} / \mathrm{dl}$ ou ferritina $<15 \mathrm{mcg} / \mathrm{dl}$. A anemia macrocítica foi diagnosticada quando os níveis de hemoglobina estavam abaixo de $11 \mathrm{mg} / \mathrm{dll}$, associada ao aumento do volume corpuscular médio (VCM > 100 fll) e déficit de vitamina B12 e/ou ácido fólico.

Como anemia grave considerou-se $\mathrm{Hb}<8 \mathrm{mg} / \mathrm{dl}$. As pacientes que na propedêutica inicial apresentaram anemia grave, $\mathrm{Hb}<8 \mathrm{mg} / \mathrm{dl}$, foram cadastradas no baixo risco e encaminhadas ao Alto Risco Obstétrico (ARO). A ferritina foi realizada em todas as pacientes com hemoglobina baixa, para a caracterização da anemia ferropriva. O tratamento foi realizado conforme protocolo dos serviços, considerando a anemia diagnosticada.

Os dados foram digitados diretamente em planilha Excel e depois exportados para o Epi Info vs 6.0. Os testes estatísticos utilizados foram a análise da variância (ANOVA) para comparação de médias entre grupos ou teste qui-quadrado para variáveis categóricas ou, quando necessário, o teste de Kruskall Wallis (Teste H). O nível de significância foi $p<0,05$. O presente estudo foi encaminhado via Plataforma Brasil e aprovado pela Comissão de Ética da Faculdade de Medicina de Barbacena (Parecer de número 31882519.4.0000.8307).

\section{RESULTADOS}

Foram analisados 293 dados de gestantes adolescentes. Estes dados foram comparados com 1121 pacientes adultas de baixo risco, totalizando 1414 gestantes atendidas nesse período. Na Tabela 1 são apresentados os antecedentes obstétricos e dados epidemiológicos das pacientes estudadas.

Na Tabela 2 são apresentados os dados clínicos das pacientes estudadas, comparando as pacientes adolescentes com as adultas. Observou-se que as pacientes adolescentes tiveram maior frequência de corrimento vaginal $(p=0,02)$, ITU alta $(p=0,02)$ e amniorrexe prematura $(p=0,002)$. 
Tabela 1 - Antecedentes obstétricos e dados epidemiológicos das pacientes estudadas.

\begin{tabular}{|c|c|c|c|c|c|c|c|c|c|c|}
\hline Pacientes & Idade & Gesta & Para & Etilismo & Tabagismo & RF & PN & MCP & IRS & IGRAV \\
\hline Adolescentes & $17,2( \pm 2,03)$ & $1,28( \pm 0,62)$ & $0,70( \pm 0,51)$ & $6,4 \%$ & $7,80 \%$ & $\begin{array}{c}\mathrm{R} \$ 1225,00 \\
( \pm \mathrm{R} \$ 842,00)\end{array}$ & $20,40 \%$ & $46,20 \%$ & $14,7( \pm 2,03)$ & $16.60( \pm 2,38)$ \\
\hline $\begin{array}{l}\text { Adultas de } \\
\text { baixo risco }\end{array}$ & $28,5( \pm 6,70)$ & $2,62( \pm 2,62)$ & $1,30( \pm 1,42)$ & $9,4 \%$ & $12,66 \%$ & $\begin{array}{c}\mathrm{R} \$ 1386,00 \\
( \pm \mathrm{R} \$ 934,00)\end{array}$ & $79,60 \%$ & $53,80 \%$ & $16,9( \pm 3,18)$ & $21,3( \pm 5,43)$ \\
\hline Valor de $p$ & $<0,001$ & $<0,001$ & $<0,001$ & 0,22 & 0,02 & 0,007 & $<0,001$ & 0,147 & $<0,001$ & $<0,001$ \\
\hline
\end{tabular}

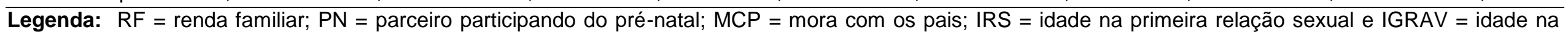
primeira gravidez. Fonte: Guia LCM, et al., 2021.

Tabela 2 - Comparação entre os dados clínicos entre pacientes adolescentes e adultas.

\begin{tabular}{|c|c|c|c|}
\hline Variáveis clínicas & Adolescentes & Adultas & Valor de $p$ \\
\hline \multicolumn{4}{|l|}{ Corrimento vaginal } \\
\hline Sim & 21 & 46 & \multirow{2}{*}{0,02} \\
\hline Não & 272 & 1075 & \\
\hline \multicolumn{4}{|l|}{ ITU alta } \\
\hline Sim & 15 & 28 & \multirow{2}{*}{0,02} \\
\hline Não & 278 & 1093 & \\
\hline \multicolumn{4}{|l|}{ ITU baixa } \\
\hline Sim & 30 & 105 & \multirow{2}{*}{0,651} \\
\hline Não & 263 & 1016 & \\
\hline \multicolumn{4}{|l|}{ CIUR } \\
\hline Sim & 4 & 6 & \multirow{2}{*}{0,13} \\
\hline Não & 289 & 1115 & \\
\hline \multicolumn{4}{|c|}{ Amniorrexe prematura } \\
\hline Sim & 2 & 0 & \multirow{2}{*}{0,006} \\
\hline Não & 2 & 0 & \\
\hline \multicolumn{4}{|c|}{ Peso estimado fetal a partir de 36 semanas (média) } \\
\hline & $2998,70( \pm 33,9)$ & $3023,20( \pm 35,1)$ & 0,68 \\
\hline \multicolumn{4}{|c|}{ Altura uterina materna a partir de 36 semanas (média) } \\
\hline & $33,9( \pm 2,14)$ & $35,1( \pm 14,0)$ & 0,09 \\
\hline
\end{tabular}

Fonte: Guia LCM, et al., 2021. 
A anemia ferropriva foi identificada por $98 \%$ e a macrocítica foi identificada em $2 \%$ dos casos. A média de hemoglobina nas gestantes adolescentes foi de $11,7( \pm 1,37)$ no primeiro, $11,30( \pm 1,09)$ no segundo e 11,50 $( \pm 1,52)$ no terceiro trimestres. Nas não adolescentes, a média de hemoglobina foi de $12,10( \pm 1,25), 11,50$ $( \pm 1,12)$ e $11,80( \pm 1,40)$. A média de hemoglobina foi maior nas pacientes adultas. Para o primeiro trimestre 0 valor de $p$ foi $<0,001$; para o segundo trimestre $p=0,01$ e no terceiro trimestre, $o$ valor de $p=0,05$, conforme Figura 1.

Figura 1 - Comparação entre as médias de hemoglobina (hb) nos três trimestres gestacionais

A

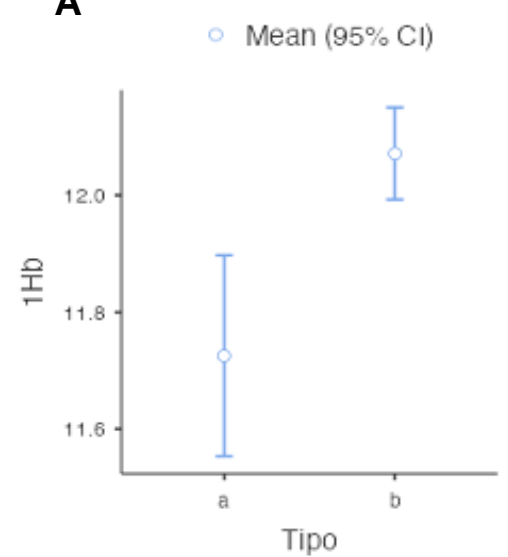

B

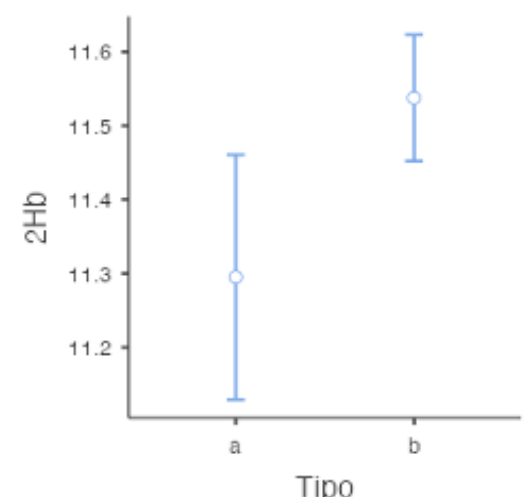

C

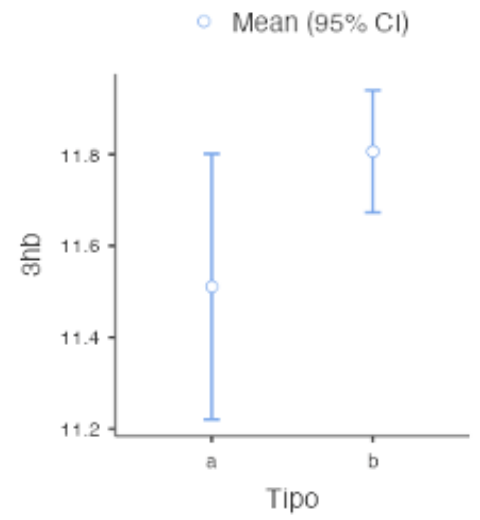

Legenda: $\mathrm{Em} \mathrm{A}$, observa-se média de $\mathrm{Hb}$ no primeiro trimestre; em $\mathrm{B}$ observa-se a média da $\mathrm{Hb}$ no segundo trimestre e em $\mathrm{C}$ a média da $\mathrm{Hb}$ no terceiro trimestre. Tipo $\mathrm{a}=$ adolescentes; Tipo $\mathrm{b}=$ adultas. Mean = média. $1 \mathrm{Hb}=$ Hemoglobina no primeiro trimestre. $2 \mathrm{Hb}=$ Hemoglobina no segundo trimestre. $3 \mathrm{Hb}=$ Hemoglobina no terceiro trimestre. Fonte: Guia LMC, et al., 2021.

A variação do hematócrito de acordo com os trimestres foi de $35,20( \pm 4,06), 34,2( \pm 3,04)$ e $34,80( \pm 3,93)$ no primeiro, segundo e terceiro trimestres para as adolescentes. Considerando as adultas, a média do hematócrito foi de $36,90( \pm 14,70), 35,40( \pm 14,50)$ e $36,4( \pm 15,3)$ respectivamente para o primeiro, segundo e terceiro trimestres. Em todos os trimestres gestacionais, o hematócrito foi menor nas pacientes adolescentes, mas sem significância estatística $(p>0,05)$.

A frequência de anemia foi calculada nos três trimestres gestacionais, sendo de $20 \%$ no primeiro, $29,4 \%$ do segundo e 18,2\% no terceiro trimestre. Na Figura 2, são apresentadas as frequências de anemia em cada trimestre, considerando adolescentes e adultas. $A$ anemia foi mais frequente em gestantes adolescentes $(\mathrm{RR}=$ $1,93 ; \mathrm{IC}=1,40-2,66 ; \mathrm{p}<0,001)$, no primeiro trimestre. No segundo trimestre, não houve diferença entre as gestantes $(p=0,142 ; I C=0,91-1,81)$, bem como no terceiro trimestre $(p=0,86 ; I C=0,60-1,54)$.

Figura 2 - Prevalência de anemia nas pacientes adolescentes e adultas e incidência trimestral de anemia no mesmo grupo.

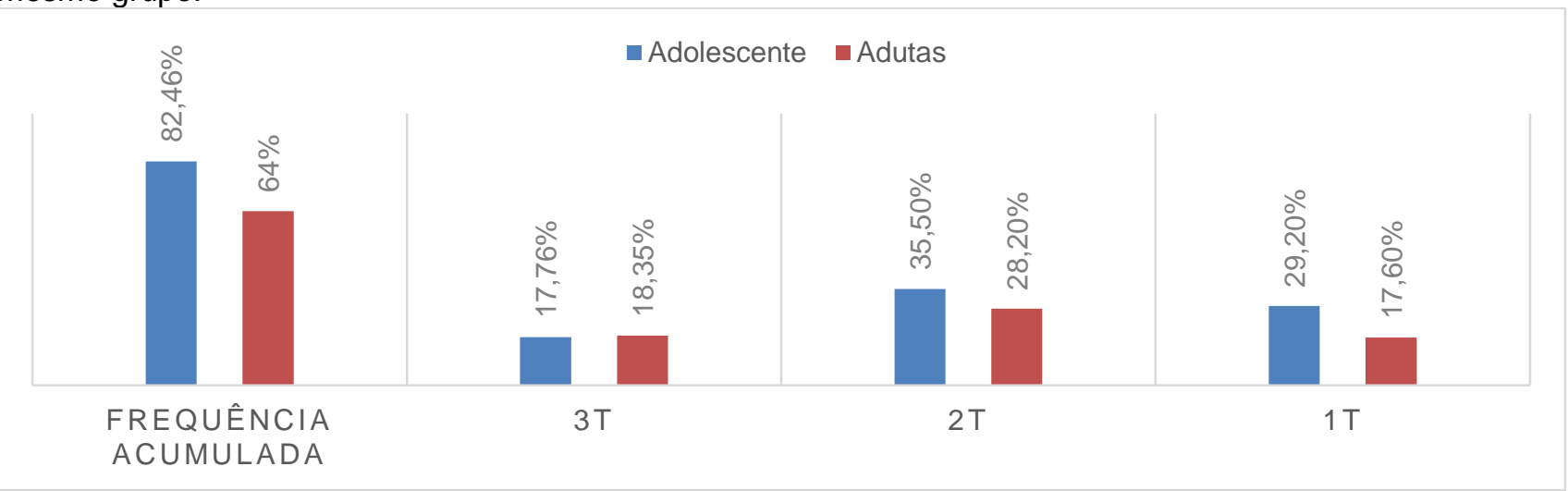

Legenda: $\mathrm{T}=$ trimestre; $3 \mathrm{~T}=$ terceiro trimestre; $2 \mathrm{~T}=$ segundo trimestre; $1 \mathrm{~T}=$ primeiro trimestre.

Fonte: Guia LMC, et al., 2021. 
A evolução da anemia ao longo dos trimestres gestacionais está representada na Figura 3. Observa-se que no segundo trimestre identificou-se maior frequência de anemia. Entretanto, houve diminuição tanto de casos novos no terceiro trimestre, como da persistência da anemia.

Figura 3 - Evolução da anemia na gravidez, com persistência e novos casos ao longo dos trimestres gestacionais.

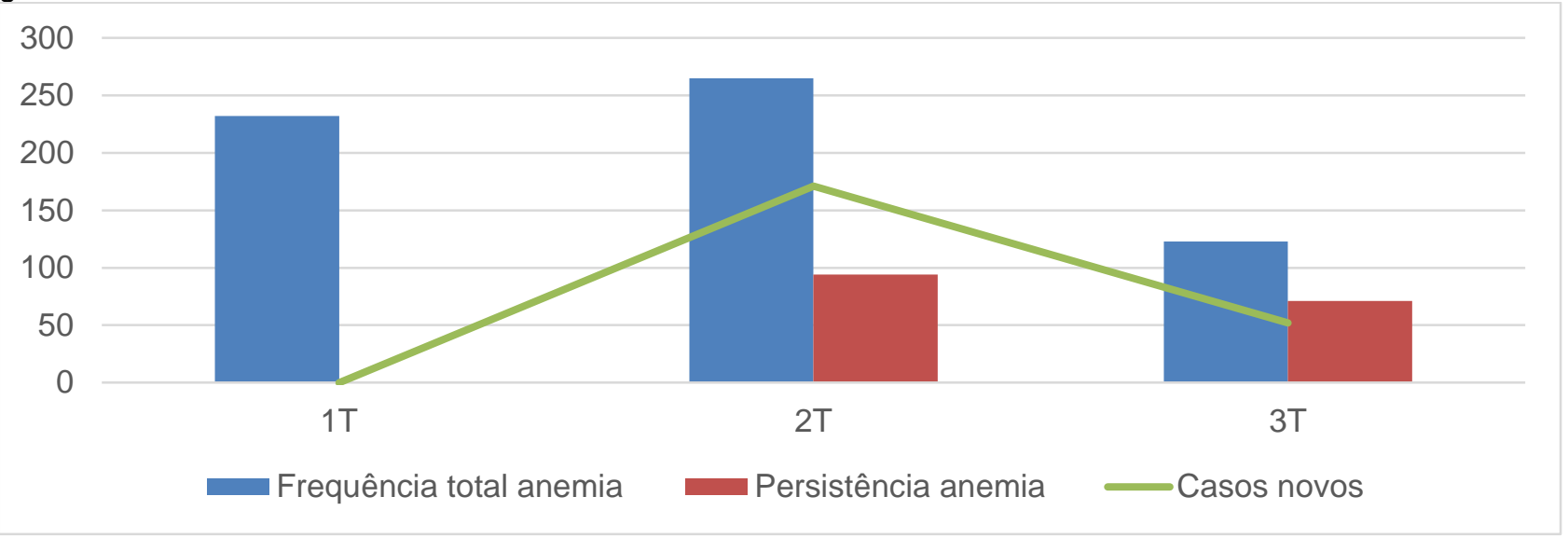

Legenda: $\mathrm{T}=$ trimestre; $3 \mathrm{~T}=$ terceiro trimestre; $2 \mathrm{~T}=$ segundo trimestre; $1 \mathrm{~T}=$ primeiro trimestre.

Fonte: Guia LMC, et al., 2021.

Realizou-se associação entre anemia no primeiro, segundo e terceiro trimestres, através de um modelo de regressão logística, para cada trimestre gestacional. No primeiro trimestre, após a aplicação do modelo, a renda familiar $(p<0,001)$ e a adolescência $(p=0,003)$ foram as variáveis associadas à anemia. No segundo trimestre e terceiro trimestre não houve associação entre os dados epidemiológicos e anemia $(p>0,05)$. $\mathrm{Na}$ Tabela 3 são apresentadas essas associações.

Tabela 3 - Associação entre anemia no primeiro, segundo e terceiro trimestres e dados epidemiológicos das pacientes estudadas.

\begin{tabular}{lccc}
\hline $\begin{array}{l}\text { Variáveis } \\
\text { epidemiológicas }\end{array}$ & $\begin{array}{c}\text { Anemia no primeiro } \\
\text { trimestre }\end{array}$ & $\begin{array}{c}\text { Anemia no segundo } \\
\text { trimestre }\end{array}$ & $\begin{array}{c}\text { Anemia no terceiro } \\
\text { trimestre }\end{array}$ \\
\cline { 2 - 4 } & & Valor de $\mathbf{p}$ & \\
\hline Gesta & 0,94 & 0,96 & 0,50 \\
Para & 0,18 & 0,69 & 0,51 \\
Uso de drogas & 0,11 & 0,99 & 090 \\
Etilismo & 0,26 & 0,50 & 0,70 \\
Idade & 0,003 & 0,082 & 0,96 \\
Renda familiar & $<0,001$ & 0,76 & 0,05 \\
Tabagismo & 0,90 & 0,70 & 0,69 \\
\hline
\end{tabular}

Fonte: Guia LMC, et al., 2021.

Quando se associou anemia no primeiro, segundo e terceiro trimestres com as complicações no pré-natal, não se identificou associação significativa estatisticamente com corrimento vaginal $(p>0,05)$, com ITU baixa ou alta $(p>0,05)$, amniorrexe prematura $(p>0,05)$, diabetes ou pré-eclâmpsia $(p>0,05)$. Não houve associação entre peso fetal e anemia no primeiro $(p=0,77)$, segundo $(p=0,65)$ e terceiro trimestres $(p=0,69)$. Na análise multivariada se identificou que o peso fetal não foi associado a anemia no primeiro $(p=0,57)$, segundo $(p=0,49)$ e terceiro trimestre $(p=0,57)$, idade $(p=0,85)$ ou ao tabagismo, etilismo ou uso de drogas $(p>0,05)$, mas sim a altura uterina $(p=0,04)$.

\section{DISCUSSÃO}

Nesse estudo foram comparados pacientes adolescentes e adultas. Os dados epidemiológicos evidenciaram que as pacientes adultas tinham maior número de gestações, partos, abortos, maior frequência 
de parceiros participando do pré-natal. Por outro lado, as adolescentes tiveram a primeira relação sexual e gravidez com idade menor, quando comparadas às adultas. A verdade é que a idade de iniciação sexual vem diminuindo ao longo dos anos, expondo-as às doenças sexualmente transmissíveis, gravidez indesejada e a complicações dessas práticas (CABRAL CS e BRANDÃO ER, 2020).

Recente estudo que avaliou a prática do casamento e gravidez precoce na Etiópia, identificou prevalência de anemia entre adolescentes do sexo feminino de 23,8\% (IC 95\%; 22,3-25,2). O estado civil (OR = 1,53, IC $95 \%=1,06-2,02)$ e engravidar ( $O R=2,80, I C$ 95\% 1,25-6,29) foram associados positivamente com anemia entre adolescentes do sexo feminino. Por isso, os autores descrevem a necessidade de políticas e programas eficazes para acabar com a prática do casamento infantil precoce e a consequente gravidez na adolescência na Etiópia (TIRUNEH FM, et al., 2021). Além disso, as adolescentes parecem apresentar maior frequência de complicações na gravidez, como, por exemplo, prematuridade, baixo peso ao nascer e menor índice de Apgar (TSHAKWENI N, et al., 2020).

Durante a gestação, o aumento da massa dos eritrócitos é menor que a expansão volumétrica do plasma, resultando em redução dos níveis de hemoglobina e hematócrito, com consequente diminuição da viscosidade sanguínea, especialmente durante o segundo trimestre. Nos casos de gravidez avançada, a recuperação dos níveis de hemoglobina se dá porque o plasma se estabiliza, ao mesmo tempo em que a massa de hemoglobina continua se elevando. Entretanto, a anemia é um evento comum na gravidez e, portanto, conhecer as alterações fisiológicas do organismo materno são importantes para o correto diagnóstico (GANDHI MH e GUPTA V, et al., 2021; ZIMMERMMANN JB, et al., 2018).

A anemia mais frequente foi a ferropriva, representada pelo decréscimo da hemoglobina, associada à diminuição do volume globular (anemia microcítica) e pela diminuição das reservas de ferritina. A ferritina sérica é o teste inicial mais específico para o diagnóstico de deficiência de ferro em mulheres grávidas e sua concentração pode estar elevada na inflamação e o uso de outros marcadores para apoiar o diagnóstico de Anemia por deficiência de ferro (ADF) pode ser necessário. Uma saturação de transferrina diminuída ou uma concentração elevada do receptor de transferrina solúvel no soro em uma paciente grávida que tem uma concentração de ferritina sérica normal ou elevada pode ser útil na confirmação da anemia ferropriva (PATEL P e BALANCHIVADZE N, 2021).

Neste estudo a frequência de anemia foi calculada nos três trimestres gestacionais, sendo mais frequente no segundo trimestre. Recente estudo também identificou maior prevalência de anemia no $2^{\circ}$ trimestre $(14,7 \%)$ (LI L, et al., 2018). A verdade é que as adolescentes acabam iniciando o pré-natal mais tardiamente e isso pode ser o responsável pela maior chance de complicações (TSHAKWENI N, et al, 2020).

Em todos os trimestres gestacionais, a média de hemoglobina e hematócrito foi menor nas pacientes adolescentes, coincidindo com informações contidas na literatura, que afirmam que ter menos de 20 anos de idade aumenta as chances de anemia e ter mais de 34 anos aumenta as chances de $\mathrm{Hb}>13,0 \mathrm{~g} / \mathrm{L}$ (RIBOT B, et al., 2018). Estes resultados são comparáveis a recente estudo que descreveu que a gravidez na adolescência foi associada riscos mais elevados de eclâmpsia $(O R=1,87 ; I C=1,57-2,23)$, anemia grave (OR $=1,18$; IC $95 \% 1,09-1,28)$, near miss materno $(O R=1,24,95 \%$ IC $1,12-1,37)$ e pequenos para a idade gestacional (PIG = 1,30, IC 95\% 1,28 - 1,33) (YANXIA X, et al., 2021).

Entretanto, nossos resultados não demonstraram associação entre anemia e resultados obstétricos desfavoráveis. Quando se avaliou as complicações neonatais, representadas pelo peso ao nascer, não se identificou diferenças entre o peso dos recém-nascidos de gestantes adolescentes e adultas, mesmo as adolescentes tendo apresentado maior frequência de anemia. Segundo estudo realizado na Venezuela, o peso ao nascer dos recém-nascidos de mães anêmicas diminuiu 12,39\% quando comparados com as gestantes sem anemia (2.970 $\pm 0,43 \mathrm{~g}$ vs. $3.390 \pm 0,32 \mathrm{~g} ; \mathrm{p}<0,0001)$ (MACHADO JRU, et al., 2015).

Creditamos os resultados positivos deste estudo a uma equipe treinada, suplementação de ferro rotineira e tratamento adequado da anemia carencial que determinou menor número de casos novos e diminuição da persistência de anemia. A ingestão nutricional de ferro é a chave para prevenir a deficiência de ferro. Acredita- 
se que a deficiência de ferro durante o primeiro trimestre tenha impacto mais negativo no crescimento fetal do que a anemia que se desenvolve mais tarde na gravidez. Em nosso serviço, todas as pacientes recebem dose profilática de ferro elementar na dosagem de $60 \mathrm{mg} /$ dia (ZIMMERMMANN JB, et al., 2018).

Quando diagnosticada anemia ferropriva, o tratamento é iniciado por via oral, com dosagem de até 200 $\mathrm{mg} /$ dia de ferro elementar. O incremento de $1 \mathrm{~g}$ nos níveis da hemoglobina são considerados satisfatórios e com boa resposta ao tratamento oral. Caso contrário, faz-se a reposição venosa. Acredita-se que a profilaxia rotineira e o tratamento adequado foram também importantes na redução da persistência da anemia e na diminuição de novos casos (PATEL P e BALANCHIVADZE N, 2021).

Entretanto, apesar de não identificarmos baixo peso ao nascer, incremento de infecções urinárias ou ginecológicas nas pacientes anêmicas, estes resultados nos preocupam, pois mostram um incremento da anemia quando comparado com estudo realizado no mesmo serviço, em 2009. Naquela época, identificou-se $2,8 \%$ de gestantes anêmicas no primeiro, $7,14 \%$ no segundo e $9,6 \%$ no terceiro trimestres de gestação. (TOTTI HKB et al, 2009).

Atualmente, a frequência de anemia triplicou e os dados epidemiológicos associados foram a baixa renda e a idade, sendo extremamente significativo se avaliarmos o quanto a pobreza aumentou em nosso país. Em 2011, o governo federal lançou o Plano Brasil Sem Miséria (PBSM) visando acabar com a pobreza extrema em nosso país, atingindo uma parcela da população que é mais vulnerável, abandonada e distante dos programas existentes (TRONCO GB e RAMOS MP, 2017).

Apesar disso, houve incremento de novos pobres, com queda do bem-estar geral e aumento da desigualdade social. Além disso, os indicadores atuais mostram que a pobreza aumentou, especialmente com a Pandemia pelo Coronavírus, sendo que a queda da renda no grupo mais pobre foi de $20.81 \%$ (NERI MC, 2021). Outro aspecto relevante é que os grupos sociais perdedores foram os analfabetos, indígenas e os jovens. Não se pode esquecer que as adolescentes fazem parte desse grupo jovem extremamente prejudicado economicamente na Pandemia (NERI MC, 2020). Sendo assim, é lícito supor que a pobreza pode ter incrementado a anemia nesse grupo populacional. Tal suposição tem apoio em estudo realizado em Ghana com gestantes adolescentes que identificou que as participantes que tiveram a experiência de "sentir fome" tiveram $3 x$ mais chance de ter estoques de ferro em depleção quando comparados com aquelas que não passaram por essa situação (ANNAN RA, et al., 2021).

Entretanto, a anemia na gravidez é um problema mundial, mas nações mais pobres sofrem mais com o problema. Na China, identificou-se anemia em $23,5 \%$ das gestantes avaliadas (LIN L, et al., 2018). Ribot B, et al. (2018) identificaram aumento da frequência de anemia, ao longo dos trimestres gestacionais, sendo $3,8 \%$ no primeiro trimestre e $21,5 \%$ no terceiro trimestre.

No Irã identificou-se prevalência de anemia geral 7,9\%, com correlação positiva entre as concentrações séricas de $\mathrm{Hb}$ e a ingestão média diária de proteína e ferro $(p<0,001)$, com os níveis de ferritina sérica e o índice de massa corporal $(p<0,001)$ e as associações inversas com número de gestações e filhos $(p<0,001)$, bem como o número de membros que compõem a família $(p<0,05)$ (ASGHARI $S$, et al., 2020).

No Brasil, estudo realizado na Paraíba, verificou que 12,5\% das gestantes estavam em estado de desnutrição, $37,5 \%$ em eutrofia e $50 \%$ com excesso de peso na primeira consulta. Além disso, $29,2 \%$ dos recém-nascidos apresentaram peso insatisfatório e baixo peso (FREITAS HBM, et al., 2018). Importante ressaltar ainda que o Brasil é um país heterogêneo, com áreas mais ricas, desenvolvidas, com maior acesso e melhor qualidade de vida, e outras realmente mais pobres, o que pode dificultar o estabelecimento de estratégias para a medição da pobreza e articulação de programas sociais. Entretanto, é notório que não possuir meios de garantir a própria alimentação diária mínima é indicativo de pobreza e as repercussões econômicas são vistas diariamente nos consultórios médicos, sendo a anemia um dos sinais-sintomas identificados (TRONCO GB e RAMOS MP, 2017).

Desta forma, acredita-se que políticas públicas voltadas para as adolescentes, desde a orientação sexual até o cuidado pré-natal, devam ser otimizadas para que as complicações da gestação não aumentem com a baixa condição socioeconômica e cultural. 


\section{CONCLUSÃO}

Os resultados deste estudo mostraram que a anemia é um evento frequente na população obstétrica. Entretanto, as mulheres mais jovens, adolescentes, oram as mais afetadas, bem como aquelas com menor renda familiar. Sendo assim, sugere-se que políticas públicas de saúde devam ser implementadas especificamente para as adolescentes, para minimizar os possíveis resultados obstétricos adversos, já que elas também apresentaram maior frequência de amniorrexe prematura, corrimentos vaginais e infecções urinárias. Além disso, não se consegue desvincular a saúde da baixa condição econômica, sendo assim, cuidar da população vulnerável com políticas econômicas específicas é um dos meios de melhorar a condição de saúde da população.

\section{REFERÊNCIAS}

1- ANNAN RA, et al. Factors associated with iron deficiency anaemia among pregnant teenagers in Ashanti Region, Ghana: A hospital-based prospective cohort study. PLoS One, 2021; 16(4): e0250246.

2- ANDRADE TR e CAIXETA BT. Prevalência de anemia em gestantes adolescentes de unidades básicas de saúde de um município no interior de Minas Gerais. Revista de Psicologia e Saúde em Debate, 2018; 4: 92.

3- ASGHARI S, et al. Prevalence and predictors of iron-deficiency anemia: Women's health perspective at reproductive age in the suburb of dried Urmia Lake, Northwest of Iran. J Educ Health Promot, 2020; 29; 9: 332.

4- BOMFIM VVBS, et al. Repercussões da deficiência de ferro durante a gestação e puerpério para o binômio maternofetal. REAS, 2020; 12(2): e5154.

5- BRASIL. Ministério da Saúde. Programa Nacional de Suplementação de Ferro. 2013. Disponível em: https://bvsms.saude.gov.br/bvs/publicacoes/manual_suplementacao_ferro_condutas_gerais.pdf. Acessado em 21 de maio 2021.

6- FREITAS HBM. A influência do estado nutricional durante o período gestacional e sua correlação no peso do recémnascido. REAS, 2019; 19:e206.

7- GANDHI MH, GUPTA V. Physiology, Maternal Blood. StarPearls [interne]. Treasure Island (FL): StarPearls Publishing. 2021:30

8- JARAMILLO-MEJÍA MC, HERNICHOVSKY D. Early adolescent childbearing in Colombia: time-trends and consequences. Cadernos de Saúde Pública, 2019; 35(2): e00020918

9- JUGHA VT, et al. Dietary Diversity and its Contribution in the Etiology of Maternal Anemia in Conflict Hit Munt Cameroon Area: A Cross Sectional Study. Front Nutr, 2021; 7: 1-11.

10-KARA WSK, et al. Anaemia in pregnancy in Southern Tanzania: Prevalence and associated risk factors. Afr J Reprod Health, 2020; 24(3): 154-160

11-KARAÇAM Z, et al. The impact of adolescent pregnancy on maternal and infant health in Turkey: Systematic review and meta-analysis. J Gynecol Obstet Hum Reprod, 2021; 50(4): 102093

12-LIN L, et al. Prevalence, risk factors and associated adverse pregnancy outcomes of anaemia in Chinese pregnant women: a multicentre retrospective study. BMC Pregnancy Childbirth, 2018; 18(1): 111.

13-MACHADO JRU, et al. Anemia materna y peso al nascer em produtos de embarazos a término. Rev. Chil. Obstet. Ginecol, 2015; 80 (4): 297-305

14-NERI MC. Bem-Estar Trabalhista, Felicidade e Pandemia - Sumário Executivo. 2021. 17p.

15-NERI MC. Efeitos da Pandemia sobre o mercado de trabalho brasileiro: Desigualdades, ingredientes trabalhistas e o papel da jornada. 2020. $15 p$.

16-OMS. Organização Mundial de Saúde. World Health Organization. The global prevalence of anaemia in 2011. Geneva:

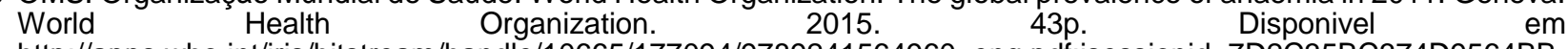
http://apps.who.int/iris/bitstream/handle/10665/177094/9789241564960_eng.pdf;jsessionid=7D2C35BC374D9564BB 4F1DDD32914955? sequence=1. Acessado 21 maio 2021.

17-PARKS S, et al. Maternal anaemia and maternal, fetal, and neonatal outcomes in a prospective cohort study in India and Pakistan. BJOG, 2019; 126 (6): 737-43.

18-PATEL P, BALANCHIVADZE N. Hematologic Findings in Pregnancy: A Guide for the Internist. Cureus, 2021; 13(5): e15149.

19-RIBOT B, et al. Prevalence of anaemia, risk of haemoconcentration and risk factors during the three trimesters of pregnancy. Nutr Hospm, 2018; 35(1): 123-130.

20-SUN D, et al. Anemia in Pregnancy: A Pragmatic Approach. Obstet Gynecol Surv, 2017; 72(12): 730-737.

21-TIRUNEH FN, et al. Associations of early marriage and early childbearing with anemia among adolescent girls in Ethiopia: a multilevel analysis of nationwide survey. Arch Public Health, 2021; 79(1): 91.

22-TOTTI HKB, et al. Frequência de anemia e valores de normalidade para a hemoglobina em gestantes. HU Revista, Juiz de Fora. 2009; 35(4): 282-86.

23-TSHAKWENI N, et al. A comparative analysis of teenagers and older pregnant women concerning maternal and neonatal adverse outcomes in Raymond Mhlaba sub-District, South Africa. Afr J Reprod Health. 2020 Dec;24(4):138146.

24-TRONCO GB, RAMOS MP. Linhas de pobreza no Plano Brasil Sem Miséria: análise crítica e proposta de alternativas para a medição da pobreza conforme metodologia de Sonia Rocha. Revista de Administração Pública, 2017; 51(2): 294-311.

25-YANXIA XIE, et al. Characteristics and adverse outcomes of Chinese adolescent pregnancies between 2012 and 2019. Scientific Reports, 2020; 11: 12508

26-ZIMMERMMANN JB, et al. Condutas em Obstetrícia para gestantes de baixo risco: protocolo do serviço de Obstetrícia da Faculdade de Medicina da UFJF. Bragança Paulista: Margem da Palavra, 2018. 